\title{
A Review of Hydride Precipitates in Titanium and Zirconium Alloys: Precipitation, Dissolution and Crystallographic Orientation Relationships
}

\author{
Egle CONFORTO*, Xavier FEAUGAS \\ LaSIE, La Rochelle University, Avenue Michel Crépeau, 17042 CEDEX 1, La Rochelle, France \\ *egle.conforto@univ-lr.fr
}

\begin{abstract}
$\underline{\text { Abstract }}$
This work proposes a review of recent results on the formation and dissolution of hydrides in HCP alloys (Ti and Zr alloys) correlated to the nature of crystallographic hydride phases and their ORs. The crystallographic coherence observed between the surface hydride layer and the substrate is very important for many applications as for biomaterials devices. Five particular orientation relationships (OR) were identified between titanium/zirconium hydride precipitates and the $\alpha$-Ti and $\alpha$-Zr substrates. In addition, the nature of hydrides have a large implication on the ductility, the strain hardening, and the local plastic strain accommodation in the Ti alloys. Our studies using XDR, TEM and SEM-EBSD have been demonstrating that the nature of the hydride phase precipitates depends on the hydrogen content. DSC has been used to obtain the hydride dissolution and precipitation energy values at the bulk scale, whose difference can be associated to misfit dislocations. Local in-situ TEM dissolution observations show the depinning of part of misfit dislocations during dissolution process. Hydride reprecipitation is thus possible only if hydrogen is not driven away during heating by misfit dislocations depinning.
\end{abstract}

\section{Introduction}

Hydrogen diffuses very rapidly into materials with close-package hexagonal (HCP) structure as titanium, zirconium and their alloys. As long as $\mathrm{Ti}-\alpha$ and $\mathrm{Zr}-\alpha$ accept hydrogen in solid solution, it is randomly distributed to energetically favorable tetrahedral sites $[1,2]$. The equilibrium phase diagrams of $\mathrm{Ti}-\alpha$ and $\mathrm{Zr}-\alpha$ show that $\mathrm{H}$ solubility is very low at ambient temperature, which leads to the spontaneous precipitation of hydride phases $\mathrm{TiH}_{\mathrm{x}}$ and $\mathrm{ZrH}_{\mathrm{y}}$ [3]. This phase transformation has a technological importance, because it generates a volume expansion (around 15\%) which induces high internal stresses [4,5] at the origin of a decreased toughness [6]. Hydrogen can favor the refinement of some microstructures of Ti alloys [7], and improve the biocompatibility at the surface of biomaterials [8]. However, this can also lead to possible embrittlement shown in several industrial applications [9]. Despite of this fact, a direct correlation between damage processes and the nature of hydride need to be improved [10].

The dependence of the nature of the precipitate phases on the $\mathrm{H}$ content and on the temperature stability will define the crystallographic relationships existing between the hydride and the matrix ( $\alpha$ phase). We shall discuss these points with reference to our experimental work [11]. The importance of misfit dislocations in the accommodation of the elastic distortion between the substrates and hydride precipitates is considerable. Their role on the dissolution/reprecipitation process is will be discussed in the present review, as well as their influence on the crystallographic relationship with the substrate after many thermal cycles.

\section{The precipitation of hydrides in HCP structures}

According to Zhang and Kelly criteria [12], it is observed that the hydride precipitation is a diffusional-displacive transformation with a mixed character [13]. It is diffusive because it involves a change of composition controlled by the long-range diffusion of hydrogen. In addition, there is no shear displacement, and interfaces have low indexes. The transformation has however also displacive character, because the change in the arrangement of Ti atoms occurs in small cells, with no long-range diffusion, by the fast motion of steps.

Three different hydride phases can precipitate in hexagonal close-package HCP alloys: $\gamma, \delta$ and $\varepsilon$ whose structure and morphology depend on the hydrogen content (See Table 1). It has been demonstrated that the metastable $\gamma$-TiH phase, which precipitates in Hpoor Ti, shows long and thin lamellae of face-centered tetragonal (FCT) structure with $\mathrm{c} / \mathrm{a}>1$. The stable face-centered cubic (FCC) $\delta$-phase of $\mathrm{CaF}_{2}$ structure type develops at intermediate $\mathrm{H}$ contents. In the literature another FCT phase is reported (the $\varepsilon$ one) for which $\mathrm{c} / \mathrm{a}<1[14]$.

Various factors influence the hydride precipitation (composition, texture, work-hardening...). Intermediate/high hydrogen contents and slow cooling favor $\delta$-hydride precipitation. However, after fast cooling, the $\gamma$-hydride is often observed. The $\delta$-hydride precipitation is favored under traction or shear stress, meanwhile $\gamma$-hydride formation is observed in absence of stress [15,16]. Hydrides tend to precipitate in a plane perpendicular to the tensile direction [17-19]. In addition, the volume expansion generates important elastic strains, which play a determinant role in the transformation, and enhances the close interlocking between precipitates with different OR [13] (see next paragraph).

The content of the alloy elements influences the hydride nature $(\gamma$ or $\delta)$ [18]. In particular, Zr alloys with oxygen concentrations higher than 1000 wppm favor the precipitation of $\delta$-hydride. The precipitation sequence for hydrides of different natures can be 
different depending on factors as the total and local hydrogen content and the crystallographic orientation of the matrix. In general, the stable $\delta$-hydride is directly formed at the $\mathrm{TiH}_{\mathrm{x}} / \mathrm{Ti}$ interface for favorable crystallographic orientations or through the formation of the intermediate, $\gamma$-TiH phase [20] during the substrate H-enrichment process. The transformation from $\gamma$-TiH to $\delta$-TiHx occurs without changes in the orientation relationships with the $\alpha$-Ti matrix.

We observe that the decreasing of the hydrogen content in the $\delta$-phase associated to the hydrogen diffusion can lead to the inverse transformation: $\delta-\gamma$ [21]. This result was confirmed for global and local approaches in recent works on $\mathrm{Zr}$ alloys, where the formation of $\gamma-\mathrm{ZrH}$ at the interface of $\delta-\mathrm{ZrH}_{1.66}$ with the $\alpha-\mathrm{Zr}$ substrate was also observed [11]. This will be examined in details in the next paragraph and illustrated in Fig. 3. The $\gamma$-phase forms from the $\alpha$-phase via a shearing of the hexagonal lattice simultaneously to hydrogen atom diffusion [14]. The $\delta-\gamma$ transformation seems to follow a similar mechanism [21]. The formation of the $\delta$-phase from the $\alpha$-phase follows a transformation of a quasi-martensitic type [22].

\section{Crystallographic orientation relationship (OR) between hydride precipitates and HCP substrate}

The crystallographic coherence between the hexagonal substrate and the hydride precipitates is high with a large elastic distortion partially accommodate by misfit dislocations. The crystallographic coherence between surface layer and the substrate is very important for biomaterials of $\mathrm{Ti}$ and $\mathrm{Ti}$ alloys because it guarantees no delamination, and the expected lifetime in the human body with good mechanical performances [8,23]. In addition, the nature of the hydride has a large implication on the ductility, the strain hardening, and the local plastic strain accommodation in the titanium and zirconium alloys [4,6]. In fact, our early studies by TEM in Ti and Ti alloys for biomaterials applications by TEM showed a strong orientation relationship between the $\alpha$-Ti and the grains of the hydride layer grown at the surface of the substrate after a chemical etching [8,23-25], aiming to increase osteointegration (Fig. 1).

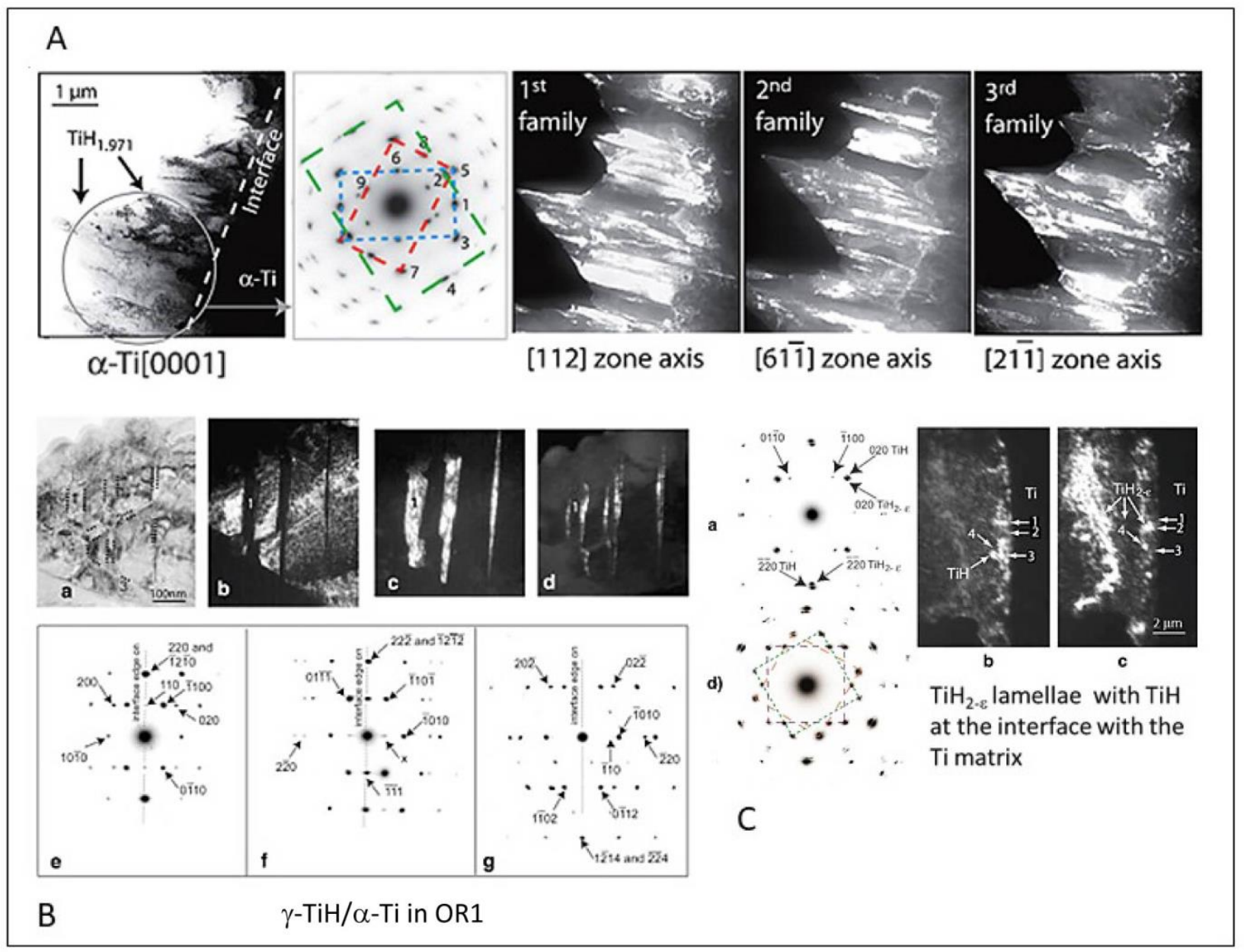

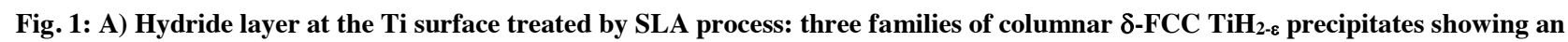
epitaxial relationship with the substrate. Deep in the bulk: B) $\gamma$-FCT TiH precipitates in OR1 with the Ti substrate; C) $\delta$-FCC TiH 2 -e precipitates with $\gamma$-FCT TiH at the interface with the Ti substrate. Both hydride phases are simultaneously in OR1 with the Ti substrate. 
Beyond the orientation relationship between the substrate and the three families of parallel columnar grains, these latter had common (220) crystallographic directions between them, as shown in Fig. 1A. Deeper in the bulk of the same sample (Fig. 1B) separated, parallel $\gamma$-TiH lamellae were found in OR1 (see definition of OR in the next paragraph) with the Ti substrate. In Fig. 1C, a $\gamma$-TiH precipitate was found at the $\delta-\mathrm{TiH}_{2-\varepsilon} / \mathrm{Ti}$ interface, both hydride phases being simultaneously in the same OR1 with the Ti substrate [20].

TEM Bright-Field (BF) images and Selected Area Electron Diffraction (SAED) patterns allowed the identification of five particular epitaxial orientation relationships (OR) [13,20, 26-29] between the HCP matrix and FCT and FCC hydrides. They were stablished following some rules [20] based on the

Edge-to-edge matching model [30]. The parallel planes, the parallel zone axes between the two phases for each OR as well as those at the interface are:

OR1 : (0001) // (001), [1̄10] // [110], interface plane $(10 \overline{1} 0) / /(2 \overline{2} 0)$

OR2 : $(0001) / /(1 \overline{1} 1)\left(\right.$ angle of $\left.4^{\circ}\right),[1 \overline{2} 10] / /[110]$, interface plane $(10 \overline{1} 3) / /(2 \overline{2} 0)$

OR3 : $(10 \overline{1} 0) / /(\overline{1} 11),[1 \overline{2} 10] / /[110]$, interface plane $(0001) / /(2 \overline{2} 4)$

OR4 : $(\overline{1} 011) / /(001),[1 \overline{2} 10] / /[110]$, interface plane $(10 \overline{1} 1) / /(\overline{1} 11)$

OR5 : $(0002) / /(\overline{2} 0 \overline{2}),[2 \overline{1} \overline{1} 0] / /[\overline{1} \overline{1} 0]$, interface plane $(0002) / /(220)$

The mechanisms of precipitation and dissolution of hydrides in HCP substrates and their crystallographic orientation relationship were studied through $\mathrm{Zr}$ substrates used in the nuclear industry. The same ORs first observed in Ti alloys were also observed between $\mathrm{Zr}$ hydrides and $\mathrm{Zr}$ substrates. EBSD analysis, which is a meso-scale technique, confirms that several hydrides can be found in the same ORs with the substrate over areas of tens square microns [16, 31]. By using EBSD as a single analysis technique [31], authors affirm that the only hydride phase found in a sample containing 147 wppm in $\mathrm{H}$ is the $\delta$ one. This $\mathrm{H}$-content falls in the range between that where $\gamma$-FCT forms $(\sim 100 \mathrm{wppm})$ and that where only $\delta$-FCC is found $(\sim 300 \mathrm{wppm})$. However, some uncertainty can occur by using EBSD to distinguish $\gamma$-FCT and $\delta$-FCC hydrides because the phase identification is based on the angles of the unitary cell. In such case, the phase identification has to be performed by electron diffraction in a TEM.

The crystallographic parameters of the hydride phases as well as the ORs they form with $\alpha$-Ti and $\alpha$-Zr are listed in Table 1 . Investigations on the existence of other ORs between hydrides and HCP substrates are in progress. Recently OR1 [16], OR3 and OR5 [11] were observed in $\delta$-hydrides/ $\alpha$-Zr. OR2 was recently identified in $\delta$-hydrides/ $\alpha$-Ti system for the T40 alloy [32].

Table 1: Characteristics of the various hydride phases in $\alpha-\operatorname{Ti}$ and $\alpha-\operatorname{Zr}[3,17,20,33-44]$

\begin{tabular}{|c|c|c|c|c|c|}
\hline Hydride & Metal & Composition & $\begin{array}{l}\text { Crystallographic } \\
\text { Parameters (nm) }\end{array}$ & $\begin{array}{l}\text { Orientation } \\
\text { relationship }\end{array}$ & $\begin{array}{l}\text { Volume } \\
\text { dilatation }\end{array}$ \\
\hline \multirow{2}{*}{$\begin{array}{c}\gamma \\
(\mathrm{FCT})\end{array}$} & $\alpha-\mathrm{Ti}$ & $\mathrm{TiH}$ & $\begin{array}{l}\mathrm{a}=0.420 \\
\mathrm{c}=0.470\end{array}$ & $\begin{array}{l}\text { OR1 } \\
\text { OR2 }\end{array}$ & 12.5 to $14 \%$ \\
\hline & $\alpha-Z r$ & $\mathrm{ZrH}$ & $\begin{array}{l}a=0.462 \\
c=0.489\end{array}$ & $\begin{array}{l}\text { OR1 } \\
\text { OR2 }\end{array}$ & 12.3 to $15.7 \%$ \\
\hline \multirow{2}{*}{$\begin{array}{c}\delta \\
(\mathrm{FCC})\end{array}$} & $\alpha-\mathrm{Ti}$ & $\begin{array}{c}\mathrm{TiH}_{\mathrm{x}} \\
1.5<\mathrm{x}<1.99\end{array}$ & $a=0.440$ & $\begin{array}{c}\text { OR1, OR2* } \\
\text { OR3, OR4 }\end{array}$ & 21 à $29 \%$ \\
\hline & $\alpha-Z r$ & $\begin{array}{c}\mathrm{ZrH}_{\mathrm{x}} \\
1.5<\mathrm{x}<1.9\end{array}$ & $a=0.478$ & $\begin{array}{l}\text { OR2,OR } 1 * \\
\text { OR3*,OR5* }\end{array}$ & 16.3 to $17.2 \%$ \\
\hline \multirow{2}{*}{$\begin{array}{c}\varepsilon \\
(\mathrm{FCT})\end{array}$} & $\alpha-\mathrm{Ti}$ & $\begin{array}{c}\mathrm{TiH}_{\mathrm{x}} \\
1.8<\mathrm{x} \leq 2\end{array}$ & $\begin{array}{l}\mathrm{a}=0.447 \\
\mathrm{c}=0.440\end{array}$ & $\begin{array}{l}\text { OR1 } \\
\text { OR3 }\end{array}$ & 3 to $14.1 \%$ \\
\hline & $\alpha-\mathrm{Zr}$ & $\begin{array}{c}\mathrm{ZrH}_{\mathrm{x}} \\
1.71<\mathrm{x} \leq 2\end{array}$ & $\begin{array}{l}\mathrm{a}=0.495 \\
\mathrm{c}=0.446\end{array}$ & & \\
\hline
\end{tabular}

*Recent results reported in $[11,16,32]$

Dislocations are necessary to accommodate the misfit between FCT and FCC hydrides and the hexagonal matrix [37]. Carpenter $e t$ al. proposes that the large shear strains associated to the precipitation mechanism may play an important role in the preferential orientation of hydrides under stress [38]. More recently other authors have quantified the density of dislocations in Zr hydride, which seems to be higher than those in the $\mathrm{Zr}$ matrix [45,46]. If it stays unclear that defects are produced in the hydrides during their growth, it is well known now that hydrides are ductile phases [10]. The impact of hydrides formation on the storage energy need further investigations.

The importance of the role played by misfit dislocations in the dissolution-reprecipitation processes was highlighted by thermodynamic investigations on a global approach (over a large density of hydrides) after a large number of thermal cycles by 
DSC (Differential Scanning Calorimetry) [11]. Results showed that both dissolution and reprecipitation temperatures increase with the number of cycles, and the dissolution temperature is higher than that of precipitation independently of the hydrogen content in the range investigated. This difference in temperature and its evolution can be discussed in terms of energy. For the first thermal cycle, dissolution and precipitation enthalpy values are $48 \mathrm{~kJ} / \mathrm{mol}$ and $20 \mathrm{~kJ} / \mathrm{mol}$ respectively [11]. These values are of the same order of those measured by other authors in different $\mathrm{Zr}$ alloys [47-50]. Additionally, the evolution of the dissolution and reprecipitation temperatures with the number of cycles suggests that both energy decreases [11]. Mathematically, the difference between both enthalpies is only dependent on the stored elastic and plastic energies. The experimental difference observed was thus attributed to misfit dislocations. Their role in the hydride reprecipitation after dissolution was confirmed by TEM in-situ measurements. Misfit dislocations were observed at the $\gamma-\mathrm{ZrH} / \mathrm{Zr}$ interface at $300 \mathrm{~K}$ before heating (Fig. 2a). The depinning of a misfit dislocation is observed at $700 \mathrm{~K}$ when the precipitate dissolution is completed (Fig. 2b). Leaving the precipitation region, misfit dislocations are probably driving away part of the $\mathrm{H}$ atoms, which are no more available to re-precipitate [51]. The local decrease in concentration of $\mathrm{H}$ atoms available to precipitate can explain the difference in energy between dissolution and precipitation after a thermal cycling for a global approach.

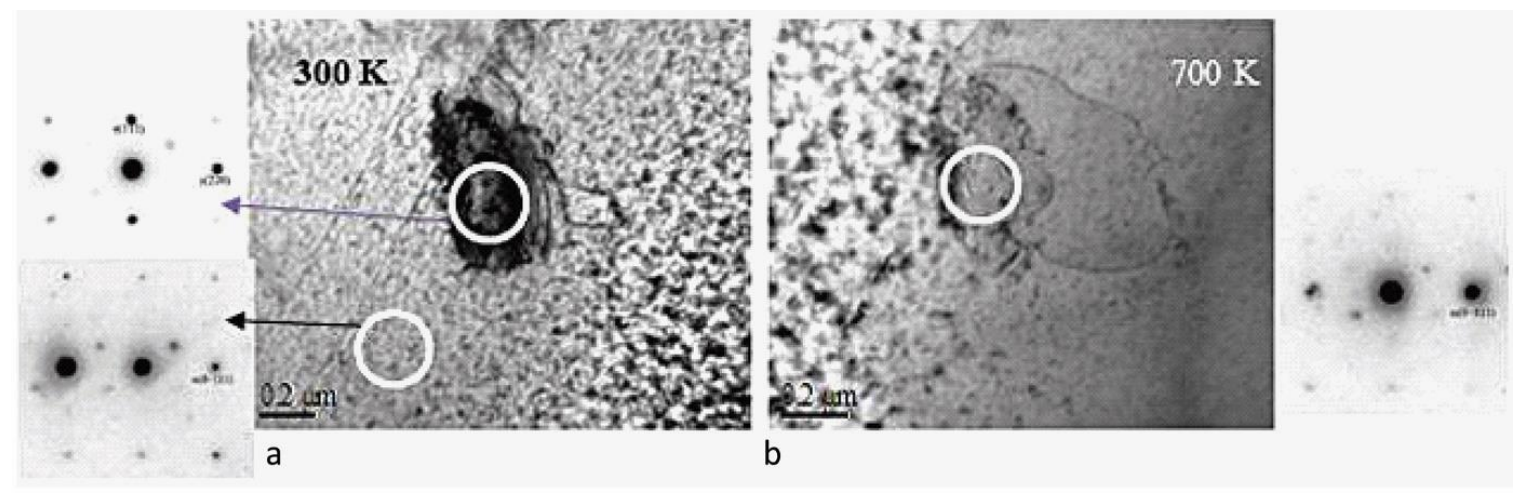

Fig. 2: A $\gamma$-hydride precipitate: (a) before and (b) after TEM in-situ dissolution, which shows a misfit dislocation depinning.

The accumulated effect of multiple misfit dislocations depinning after a large number of cycles (in a global approach, over the whole sample) can lead to the precipitation of hydrogen-poor hydride phases. In fact, the simultaneous presence of the initial hydrogenrich phase (the FCC $\delta$-hydride) and a hydrogen-poor phase (the $\gamma$-FCT) was observed at the hydride-substrate interface after cycling, as shown in Fig. 3. The reprecipitation of the $\delta$-hydride after 20 thermal cycles is still highly coherent with respect to the substrate (orientation close to an OR3 in this case) and the (220) planes of the $\gamma$-FCT phase are parallel to those of the $\delta$-FCC [57].

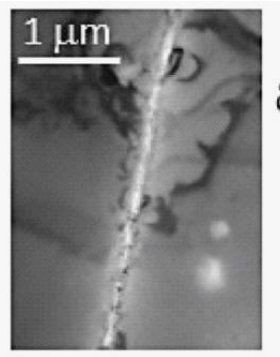

a

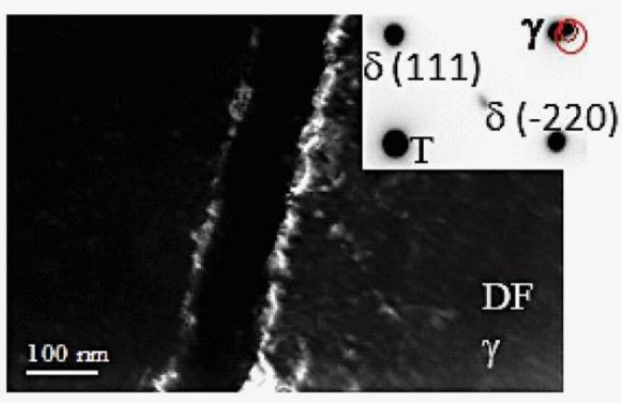

e

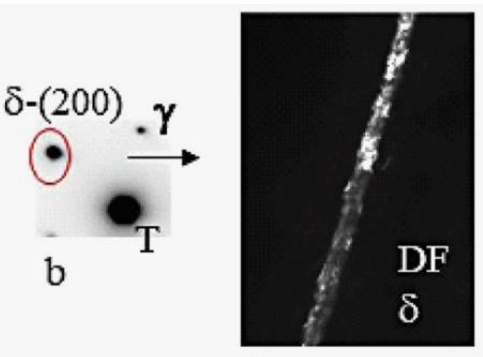

c

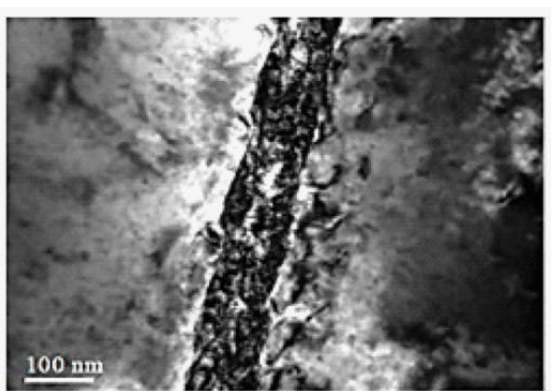

d

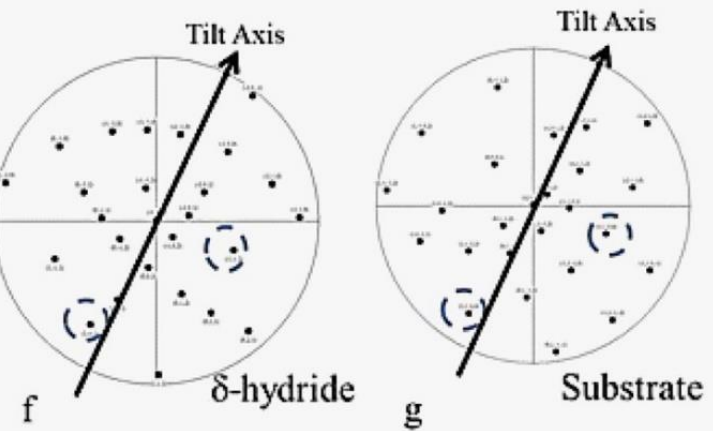

OR3 $:(-1100) / /(-111)$ and $[11-20] / /[101]$

Fig. 3: a, b, c, d) TEM images and SAED patterns showing a $\delta$-hydride in a Zircaloy-4 sample with 356 wppm in $H$ before 20 thermal cycles by DSC; $\mathrm{e}, \mathrm{f}, \mathrm{g}$ ) Simultaneous presence of $\delta$ - and $\gamma$-hydrides after cycling. 


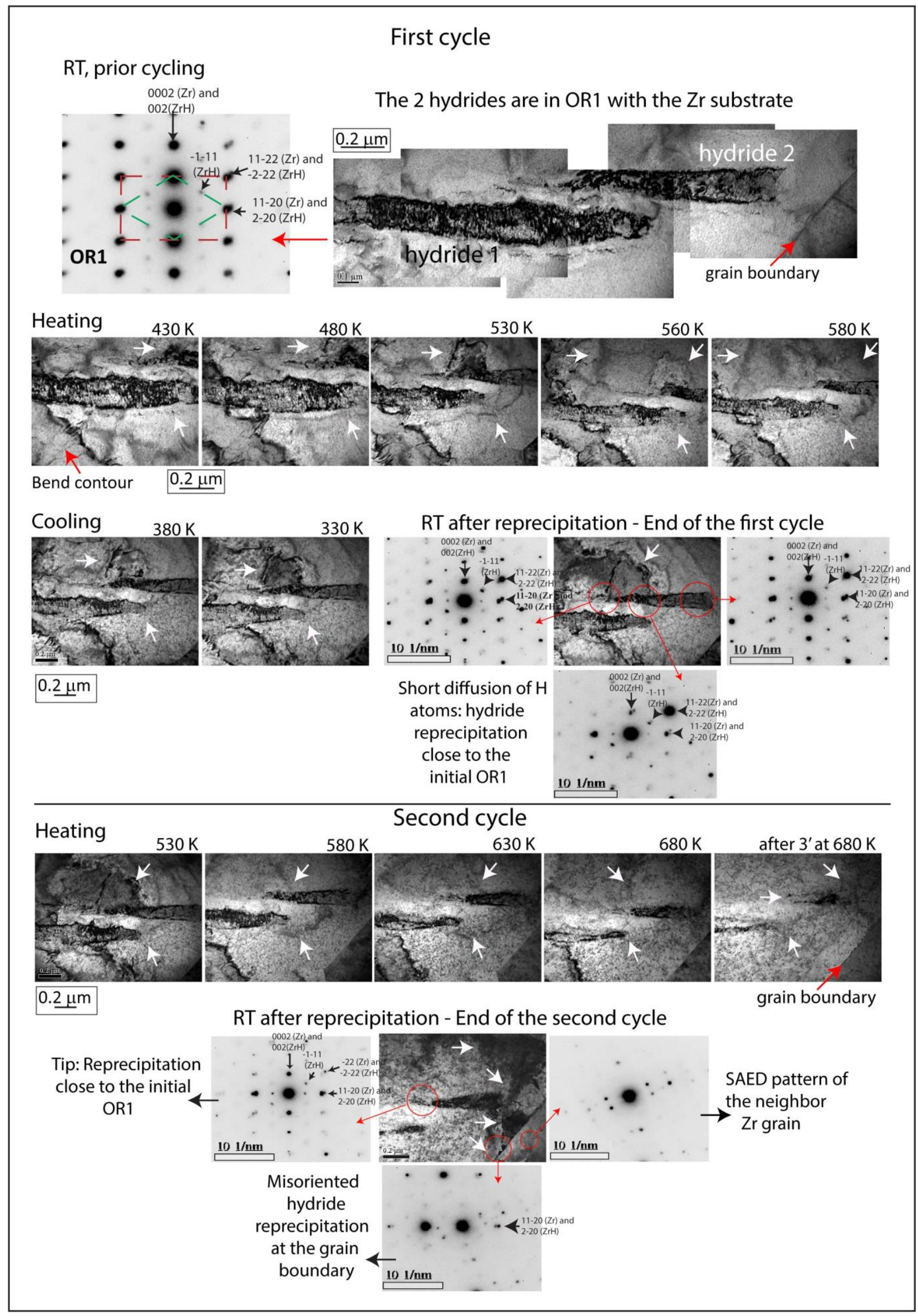

Fig. 4: Two thermal cycles between 300 and $680 \mathrm{~K}$ by TEM in-situ (images extracted from a video). After the first thermal cycle, the $\gamma$ hydride precipitate, initially in OR1, reprecipitates with a slight misorientation. At the end of the second cycle, part of $H$ atoms migrates towards a neighbor grain boundary. The hydride reprecipitation there is no more in an OR. 
These results are confirmed in a local approach by TEM in-situ in Figure 4, which shows two $\gamma$-hydrides initially in OR1 with the $\mathrm{Zr}$ substrate in a sample containing $112 \mathrm{wppm}$ in H. It was submitted to two thermal cycles between 300 and $680 \mathrm{~K}$. During the first cycle the dissolution is partial (the diffusion of $\mathrm{H}$ atoms around the regions where the precipitate is dissolved is characterized by a "dark cloud" in Fig.4, and is indicated by white arrows). After cooling, the $\gamma$-hydride reprecipitates close to the initial OR1, but slightly misoriented. During the second cycle, the hydride dissolution is almost complete. The main part of $\mathrm{H}$ atoms migrates towards a grain boundary in the neighborhood, as indicated in the figure by white arrows. The hydride reprecipitation at the grain boundary is not crystallographically oriented, as shown in the corresponding SAED pattern. Similar results were obtained for $\delta$ - $\mathrm{ZrH}_{1.66}$ precipitates in $\mathrm{Zr}$ samples containing $356 \mathrm{wppm}$ in $\mathrm{H}$. To resume, our results support the fact that misfit dislocations are fundamental in precipitation and dissolution processes. Their depinning is responsible of the decrease of the energies associated with both processes in relation with a progressive transformation of a $\gamma$-hydride (large dilatation volume) to a $\gamma$-hydride (lower dilatation volume).

\section{Conclusions:}

In a present short review of our recent results on the formation and dissolution of hydrides in HCP alloys (Ti and $\mathrm{Zr}$ alloys) we report new understanding of the nature of crystallographic hydride phases and their ORs. The hydride dissolution and precipitation energies can be associated to misfit dislocations. The depinning of part of misfit dislocations during dissolution process can modify these energies and consequently, promote a phase transformation and an irreversible process. The impact of both effects on mechanical properties need furthers investigations.

\section{$\underline{\text { References }}$}

1. R. Khoda-Bakhsh, D.K.Ross, J. Phys. F: Met. Phys. 12 (1982) 15-24.

2. C. Domain, R. Besson, A. Legris, Acta Mater. 50 (2002) 3513-3526.

3. W.M. Mueller, J.P. Blackledge, G.C. Libowitz, Metal Hydrides (1968) Academic Press, New York.

4. I. Guillot, X. Feaugas, M. Clavel, Scripta Mater. 44 (2001) 1011-1017.

5. X. Feaugas, E. Conforto, PlastOx 2007, EDP Sciences (2009) 161-178.

6. J. Huez et al., Metall. Trans. A 29 (1998) 1615-1628.

7. G. Domizzi, M.I. Luppo, G. Vigna, J. Alloy. Compd. 424 (2006) 193-198.

8. E. Conforto et al., Mat. Sci. \& Eng. C 24 (2004) 611-618.

9. B. Lustmann, F. Kerze, The metallurgy of zirconium (1955) MacGraw-Hill Book Co., New York.

10. E. Conforto, I. Guillot, X. Feaugas, Phil. Trans. R. Soc. A (2017) 375: 20160417.

11. E. Conforto et al., Mat. Sci. Forum 879 (2017) 2330-2335.

12. M.X. Zhang, P.M. Kelly, Progr. Mater. Sci. Vol. 54 (2009) 1101.

13. E. Conforto, D. Caillard, Solid State Phen. 172-174 (2011) 242-247.

14. E. Zuzek, H-Zr binary alloy phase diagram (1990) Ed. TB Massalski, ASTM Inter., 2, 2070.

15. V. Perovic et al., Acta Metall. 40 (1992) 363-372.

16. E. Conforto, X. Feaugas, Proc. PTM 2015, 231-238.

17. M.I. Luppo, A. Politi, G. Vigna, Acta Mater. 53(19) (2005) 4987-4996.

18. J. Bai, C. Prioul, D. François, Met. Mat Trans. A 25A (1994) 1185-1197.

19. C.D. Cann et al., J. Nucl. Mat. 126 (1984), 197-205.

20. E. Conforto, D. Caillard, Acta Mater. 55 (2007) 785-798.

21. J. Root et al., Acta Mater. 51 (2003) 2041-2053.

22. D.O. Northwood, U. Kosasih, Int. Met. Rev. 28 (1983) 92-121.

23. E. Conforto et al., Phil. Mag. 84(7) (2004) 631-645.

24. F.A. Müller et al. Journal of Crystal Growth 304 (2007) 464-471.

25. E. Conforto et al., Acta Biomaterialia 4 (2008) 1934-1943.

26. H. Numakura, M. Koiwa, Acta Metall. 32 (1984) 1799-1807.

27. O.T. Woo et al., Acta Metall. 33 (1985) 1897-1906.

28. O.T. Woo, G.J.C. Carpenter, Scripta Metall. 19 (1985) 931-934.

29. A. Bourret, A. Lasalmonie, S. Naka, Scripta Met 20 (1986) 861-866.

30. M.X. Zhang et al., Acta Mater. 53 (2005) 1427-1438.

31. S. Wang, F. Giuliani, T.B. Britton, Acta Mater. 169 (2019) 76-87.

32. A. Poloni et al., this conference, session T7-S1.

33. K.E. Moore, W.A. Young, J. Nucl. Mater. 27 (1968) 316-324.

34. K.G. Barraclough, C.J. Beevers, J. Nucl. Mater. 34 (1970) 125-134.

35. P.E. Irving, C.J. Beevers, Metall. Trans. A 2(2) (1971) 613-615.

36. J.S. Bradbrook, G.W. Lorimer, N. Ridley, J. Nucl. Mat. 42 (1972) 142-160.

37. G.J.C. Carpenter, J.F. Watters, R.W. Gilbert, J. Nucl. Mat. 48 (1973) 267-276.

38. G.J.C. Carpenter, Acta Metall. 26 (1978) 1225-1235.

39. G.C. Weatherly, Acta Metall. 29(3) (1981) 501-512. 
40. M.P. Puls, Acta Metall. 32(8) (1984) 1259-1269.

41. C.B. Zhang et al., Acta Metall. 44(3) (1996) 1077-1084.

42. A. Aladjem, Solid State Phen. 49-50 (1996) 281-329.

43. F.D. Manchester, Phases diagrams of binary hydrogen (2000) ASM Inter., 238.

44. T.I. Wu, J.K. Wu, Mater. Chem. Phys. 74 (2002) 5-12.

45. J-S Kim, S-D Kim, J. Yoon, J. Nucl. Mater. 482 (2016) 88-92.

46. M.A. Vicente Alvarez et al., Acta Mater. 117 (2016) 1-12.

47. Y. Fukai, The metal-hydrogen system: basic bulk properties (2005) New York, NY: Springer.

48. K. Une, S. Ishimoto, J. Nucl. Mater. 322 (2003) 66-72.

49. R. Tang, X. Yang, Int. J. Hyd. Energy 34 (2015) 7269-7274.

50. P. Vizcaino, R.O. Rios, A.D. Banchik, Thermochim. Acta 429 (2005) 7-11.

51. E. Conforto E. et al., TMS2017, The Minerals, Metals \& Materials Series (2017) F6: 771-782. 